\title{
Editorial
}

\section{Theorie der Entwicklung - Entwicklung der Theorie Post-Development und Postkoloniale Theorien als Herausforderung für eine Geographische Entwicklungsforschung}

\author{
M. Neuburger and T. Schmitt \\ Universität Hamburg, Institut für Geographie, Bundesstraße 55, 20146 Hamburg, Germany \\ Correspondence to: M. Neuburger (neuburger@geowiss.uni-hamburg.de) and \\ T. Schmitt (schmitt@geowiss.uni-hamburg.de)
}

Den eurozentrischen Charakter von Entwicklungstheorien zu kritisieren, ist wahrlich keine neue Errungenschaft innerhalb der Geographischen Entwicklungsforschung (GEF). Schon lange bevor Ulrich Menzel Anfang der 1990er Jahre laut über das Ende der Dritten Welt und das Scheitern der großen Theorie nachdachte (Menzel, 1992), bestand weitgehender Konsens darüber, dass die herkömmlichen Entwicklungstheorien bei teilweise unterschiedlichen bis diametral gegensätzlichen Analyseansätzen und dementsprechend verschiedenen Entwicklungsstrategien, eine Wohlstands- und Massenkonsumgesellschaft nach europäischem bzw. USamerikanischem Vorbild als Entwicklungsziel implizierten. In der angelsächsischen Theoriedebatte ging die Diskussion bereits in den 1980er Jahren über eine Kritik der Ziele des Entwicklungsmodells hinaus. In Anlehnung an die Postcolonial und Subaltern Studies - insbesondere beeinflusst durch die Arbeiten von Fanon, Said oder Spivak - standen die generelle Problematik der Produktion von (nicht nur akademischem) Wissen über ,,die Anderen“ und der daraus resultierenden Konstruktion von „Fremdem“ und „Eigenem“ und die Art und Weise des „Redens über“ und „Sprechens für“ im Mittelpunkt der Auseinandersetzungen. Die Development Studies interessierten sich dabei vor allem dafür, wie über einen postkolonialen Entwicklungsdiskurs koloniale Diskurse und Praktiken (re)produziert werden. In Auseinandersetzung mit der praktischen Entwicklungszusammenarbeit wurde vor allem im lateinamerikanischen Kontext die Frage diskutiert, ob das Konzept von Entwicklung an sich abzulehnen sei. Mit Bezug auf Foucaults diskurstheoretischen Ansatz wurde innerhalb der sogenannten Post-Development
(PD) Ansätze untersucht, wie mittels des Diskurses über Entwicklung bzw. Unterentwicklung das Untersuchungsobjekt konstruiert und somit Unterentwicklung erst erschaffen wird (u.a. Esteva, 1992; Escobar, 1995, aber auch Sachs, 1992; Rist, 1997). An die Stelle der Suche nach alternativen Entwicklungen rückte die Suche nach Alternativen zur Entwicklung (s. Beitrag von Ziai in diesem Heft).

Während in der angelsächsischen Development Geography spätestens seit den 1990er Jahren die Anregungen aus den Postkolonialen Theorien (PKT) und den PostDevelopment-Ansätzen aufgenommen und für die eigenen Studien produktiv gemacht wurden (Rahnema und Bawtree, 1997; Power, 2003; Radcliffe, 2005; McEwan, 2009), blieb die deutschsprachige GEF - im Gegensatz zur Neuen Kulturgeographie und der Politischen Geographie - von den Debatten erstaunlich unberührt (s. Beitrag von Lossau in diesem Heft). Erst ab den 2000er Jahren wurden die Ansätze im Kontext der deutschsprachigen GEF in mehreren Publikationen diskutiert (u.a. Korf, 2004; Graefe und Hassler, 2006; Gertel, 2007; Müller-Mahn und Verne, 2010). In der Auflage von 2007 des Lehrbuches Geographie (Gebhard et al., 2007) finden die PD-Ansätze jedoch keine Erwähnung, während die PKT lediglich innerhalb des Kapitels „Beispiele aktueller interdisziplinärer Forschungsfelder der Humangeographie“ diskutiert werden (s. Beitrag GAE_reflected in diesem Heft).

Vor diesem Hintergrund hatte sich die Tagung des Geographischen Arbeitskreises Entwicklungstheorien, die vom 29.31. Oktober 2010 in Innsbruck stattfand, die Aufgabe gestellt, sich von den Ansätzen des Post-Development und von 
den Postkolonialen Theorien irritieren zu lassen und diese für eine Geographische Entwicklungsforschung fruchtbar zu machen. Neben der Diskussion der grundsätzlichen Implikationen der Ansätze stand die Frage im Vordergrund, wie über eine akademische Wissensproduktion und über unsere eigene Forschungspraxis bestimmte Vorstellungen von Entwicklung transportiert und ungleiche Machtverhältnisse (notwendigerweise) reproduziert werden.

Zur Einführung in die Post-Development-Debatte stellt Aram Ziai in seinem Beitrag in diesem Heft den PD-Ansatz als Fundamentalkritik von Entwicklung, die als Ideologie oder „,bösartiger Mythos“ des Westens verstanden werden kann, vor. Dabei besteht der wesentliche Unterschied zu den bisherigen Ansätzen der Entwicklungsforschung darin, dass weder die mangelhafte Zielerreichung der Entwicklungsversprechen im Mittelpunkt steht, noch die gegenwärtigen Theorien besser an eine sich verändernde Wirklichkeit angepasst werden sollen. Vielmehr wird der Entwicklungsgedanke an sich ins Zentrum der Aufmerksamkeit gerückt und grundsätzlich in Frage gestellt wird. Dabei differenziert Ziai zwischen neo-populistischen und skeptischen Strömungen innerhalb des PD, erörtert ihre jeweiligen theoretischen Probleme und konzeptionellen Lücken und zeigt die Widersprüchlichkeiten und Potenziale der Alternativen zur „Entwicklung" auf.

Julia Lossau nimmt in ihrem einführenden Beitrag zu Postkolonialen Theorien die aktuellen Diskussionen in der GEF auf und betrachtet sie „durch eine postkoloniale Brille“. Dabei sucht sie nach Gemeinsamkeiten und Anknüpfungspunkten mit den PKT und deutet an, welche Impulse und Herausforderungen von einem postkolonialen Denken für die GEF ausgehen können. Auf einer paradigmatischen Ebene sieht Lossau die Notwendigkeit einer Erweiterung der grundsätzlichen erkenntnis- und wissenschaftstheoretischen Rahmung des Forschungsprozesses, insbesondere der normativen Vorannahmen und Setzungen. Zum anderen plädiert sie auf einer pragmatischen Ebene für eine Neuausrichtung der Konzeptionalisierung zentraler Begrifflichkeiten wie „Akteur“ „Gruppe“ und „Raum“. Eine zentrale Forderung an die GEF stellt aus ihrer Sicht eine grundlegende Reflexion der Einbettung der Entwicklungsforschung und der eigenen Forschungspraxis in bestehende Machtverhältnisse dar.

\section{Positionalität und eigene Positionierung}

Die Reflexion der eigenen Positionalität und der Verstrickungen in ungleiche Machtstrukturen kann jedoch die Widersprüchlichkeiten von Entwicklungsforschung bzw. Forschung an sich nicht grundlegend auflösen. Sowohl bei einer diskurstheoretischen als auch bei einer dekonstruktivistischen Herangehensweise werden die Bedingungen der eigenen Verortung und die Unmöglichkeit des Entrinnens aus den strukturellen Gegebenheiten deutlich. Diskurstheoretisch be- trachtet kann eine Verschiebung des Entwicklungsdiskurses - selbst bei einer Ablehnung des Entwicklungsbegriffes - nur aus dem Diskurs heraus bewirkt werden. Dabei besteht das grundsätzliche Dilemma darin, auf etablierte Begrifflichkeiten und Kategorien zurückgreifen zu müssen, und dadurch möglicherweise die kritisierten Zuschreibungen und Dichotomisierungen zu reproduzieren (bspw. bei der Benennung von Länderkategorien). Gleichzeitig eröffnet sich der Widerspruch, dass die Kritik an einem aufklärerischen Verständnis von Entwicklung innerhalb einer westlichen Forschungspraxis zwangsläufig immer nur eine aufklärerische Kritik sein kann. Dies bedeutet, sich gegenüber dem Widerspruch verhalten zu müssen, ,dass die Aufklärung, trotz ihrer Ausrichtung auf das weiße, männliche bürgerliche Subjekt, absolut unverzichtbar ist" (Dhawan, 2009:62).

In Anbetracht solcher Überlegungen ist es fraglich, ob das Arbeiten und Forschen von ,westlichen“ Wissenschaftler_innen in ,nicht-westlichen,, Kontexten überhaupt noch wünschenswert und möglich ist. Einen Ausweg und ein vorsichtiges „Ja“ versuchen viele postkoloniale Theoretiker_innen mit Gayatri Chakravorty Spivak's Aufforderung „unlearning one's privileges as loss“ (Spivak, 1990) zu beschreiten (s. Beiträge von Husseini de Araújo und Kersting, Schurr und Segebart und der Diskussionsbeitrag von GAEReflected in diesem Heft). Die eigenen Privilegien zu verlernen heißt jedoch nicht, sie einfach ablegen zu können. Der Prozess des Verlernens bedeutet zunächst einmal, die eigene privilegierte Position anzuerkennen. Gleichzeitig bedeutet dies die Anerkennung der Unmöglichkeit, aus den Verwobenheiten der Geschichte, aus den hegemonialen und rassistischen Macht- und Herrschaftsstrukturen, über die bestimmte Identitäts- und Sprechpositionen zugewiesen werden, herauszutreten. Verlernen bedeutet auch, unser Verständnis von Welt und Wirklichkeit in Frage zu stellen: „Verlernen muss ich auch ein Verständnis, dass das, wie ich die Realität wahrnehme, die ,Wahrheit" darstellt, denn unsere Perspektive auf die Welt ist u.a. entscheidend davon geprägt, wie wir selber als Subjekt wahrgenommen werden und welche soziale Position wir einnehmen" (Castro Varela, 2004:8). Somit gilt es, unseren Blick nicht nur zu schärfen, sondern auch zu wenden, d.h. nicht nur auf diejenigen zu schauen, die marginalisiert, unterdrückt und diskriminiert werden, da genau dieser Blick oftmals ein kolonialer Blick bleibt, der Hierarchisierungen und Dichotomien aufrecht erhält. Vielmehr gilt es, die Privilegierungs-, Normalisierungs- und Universalisierungsmechanismen in den Blick zu nehmen und Europa als silent referent zu enttarnen und zu ,provinzialisieren' (vgl. Chakrabarty, 2000; Castro Varela und Dhawan, 2009). Für die GEF heißt das, Wissenschaft als einen Ort zu benennen, an dem Wissen über die ,Anderen ' produziert wird, das nicht neutral, objektiv und unschuldig ist, sondern Standards setzt, Wirklichkeit verändert und dadurch wiederum ,anderes' Wissen zum Schweigen bringt. Nicht zuletzt verdienen auch diejenigen Mechanismen eine genauere Betrachtung, die zu einer fortschreitenden Enthistorisierung und Entpolitisierung 
der GEF und deren Wissensproduktion führen. Somit lässt sich aus den PKT auch die Forderung herauslesen, die weltweit existierenden, eklatanten sozialen Ungleichheiten (wieder) zu skandalisieren (vgl. Castro Varela, 2008:10).

\section{Forschungspraktische Konsequenzen}

Dieses starke Plädoyer für eine engagierte, sich einmischende GEF teilen letztendlich auch die verschiedenen Autor_innen dieser Ausgabe. Ein Ende der Forschung im Globalen Süden würde letztendlich zu einer eurozentrischen Nabelschau führen, die die Verwobenheiten zwischen den Gesellschaften ignoriert. Ausgehend von dieser Annahme erörtern Carolin Schurr und Dörte Segebart, wie Forschung in postkolonialen Kontexten insbesondere aus einer feministisch postkolonialen Perspektive heraus auch weiterhin möglich ist. Anhand einer reflektierten Auseinandersetzung mit ihren eigenen Forschungsmethoden und angereichert mit ganz persönlichen Einblicken in ihre Forschungspraxis geben sie Hinweise, wie die GEF auf die Herausforderungen der postkolonialen Wissenschaftskritik reagieren kann. Dabei wird deutlich, dass von den PKT keine einfachen Antworten und klaren Vorgaben für den Forschungsprozess zu erwarten sind.

Forschungspraktische Konsequenzen stehen auch im Beitrag von Shadia Husseini Araújo und Philippe Kersting im Vordergrund. Einen besonderen Stellenwert erhält ihr Beitrag, da sie nicht nur auf Erfahrungen in der Humangeographie eingehen, sondern auch die postkolonialen Implikationen eines Forschens im Globalen Süden im Hinblick auf die Physische Geographie benennen. Die Frage nach der Macht (und der Gewalt) verwendeter Begrifflichkeiten und Methoden, die Anerkennung der Beforschungsgeschichte der Beforschten, das Problem für „Andere“ zu sprechen und die Unmöglichkeit eines Forschens auf Augenhöhe sind sowohl für sozial- als auch für naturwissenschaftliche Forschungsvorhaben relevant. Dabei sehen die beiden Autor_innen eine dekonstruktivistische Vorgehensweise nur als einen ersten Schritt einer selbstreflexiven Forschungspraxis an. Mit der Metapher der Übersetzung bieten sie ein Verständnis der GEF als Scharnier zwischen postkolonialer Theorie und Forschungspraxis an, ohne dabei die sich ergebenden Widersprüche gänzlich auflösen zu wollen.

Wie PKT in der Geographie zur Anwendung kommen können, stellt Katrin Singer an einem ganz konkreten Anwendungsbeispiel vor. Anhand der Verhandlungen von Indigenität in Mexiko zeigt sie, wie Othering-Prozesse im Kontext von Nationbuilding gelesen und interpretiert werden können und welche neuen Blickwinkel sich daraus für eine GEF ergeben können. Dabei hinterfragt Katrin Singer die von der mexikanischen Dominanzgesellschaft und den indigenen Bevölkerungen machtvoll aufgeladenen Zuschreibungen und Repräsentationen und stellt sie als Ergebnis ungleicher, wechselseitiger und post-/kolonialer Verhandlungen dar.
In dem Diskussionsbeitrag des Autor_innenkollektivs GAE-Reflected, einer Gruppe Studierender, die sich vor und auf der Tagung in Innsbruck formierte, wird die aktuelle Debatte innerhalb der GEF hinsichtlich ihrer Reaktionen auf eine postkolonial inspirierte Kritik abgeklopft. In ihrem Beitrag zeichnen sie nach, wie über die Repräsentation von Wirklichkeit, u.a. an der Darstellung des Faches in einschlägigen Lehrbüchern, auch und gerade in der GEF machtgeladene Zuschreibungen vollzogen werden, die die „Anderen“ in einem „Raum von Unterentwicklung“ festschreiben. Ihren Beitrag schließen sie mit der Forderung, Machtverhältnisse und Formen der Repräsentation als entscheidende Größen innerhalb des Forschungsprozesses zu benennen und die „Postkoloniale Situation als Gegenwartsdiagnose“ anzuerkennen.

Das vorliegende Heft versteht sich somit als Stolperstein für eine geographische Wissensproduktion. Ob sich aus den Ansätzen des Post-Development und der Postkolonialen Theorien eine fruchtbare Debatte mit Anregungen für Lehre und Forschung ergeben oder ob es bei einem ,Weiter so" mit mehr oder weniger parallelen Entwicklungen bleibt, wird sich zeigen. Erste Anzeichen können bereits in der Neuauflage des Lehrbuches Geographie von 2011 ausgemacht werden, in dem den PD-Ansätzen ein eigenes kurzes Kapitel gewidmet wurde und die Bemühungen des Arbeitskreises Geographische Entwicklungstheorien für eine Bereicherung der Debatte gewürdigt werden (Gebhard et al., 2011, s. Beitrag von GAE_reflected) Letztendlich liegt die Beantwortung dieser Frage jedoch an uns und unserer Art des „GeographieMachens".

\section{Literatur}

Castro Varela, M. d. M.: Nach-Denken zum Thema „Interkulturelle Standards“ Überblick, 10, 6-8, 2004.

Castro Varela, M. d. M.: Das Begehren neu ordnen. Autonome Wissensproduktion in postkolonialer Perspektive, Frauensolidarität, 1, 10-11, 2008.

Castro Varela, M. d. M., und Dhawan, N.: Feministische Postkoloniale Theorie: Gender und (De-)Kolonisierungsprozesse. Europa provinzialisieren? Ja, bitte! Aber wie?, Femina Politica, 18, 9$18,2009$.

Chakrabarty, D.: Provincializing Europe. Postcolonial Thought and Historical Difference, Princeton UP, Princeton, N.J., 2000.

Dhawan, N.: Zwischen Empire und Empower: Dekolonialisierung und Demokratisierung, Femina Politica, 2, 52-63, 2009.

Escobar, A.: Encountering Development. The Making and Unmaking of the Third World, Princeton University Press, Princeton, 1995.

Esteva, G.: Development, in: The Development Dictionary. A Guide to Knowledge as Power, Herausgeber: Sachs, W., London, 6-25, 1992.

Gebhardt, H., Glaser, R., Radtke, U., und Reuber, P. (Hrsg.): Geographie. Physische und Humangeographie, Spektrum, München, 2007. 
Gebhardt, H., Glaser, R., Radtke, U., und Reuber, P. (Hrsg.): Geographie. Physische und Humangeographie, Spektrum, Heidelberg, 2011.

Gertel, J.: Geschichte, Struktur und fachwissenschaftliche Leitlinien der Entwicklungstheorien, in: Entwicklungsländer I. - Handbuch des Geographieunterrichts 8/I, Herausgeber: Böhn, D. und Rothfuss, E., Köln, 52-72, 2007.

Graefe, O. und Hassler, M.: Aktuelle Ansätze einer Relationalen Humangeographie in Entwicklungsländern - Einführung zum Themenheft, Geographica Helvetica, 61, 2-3, 2006.

Korf, B.: Die Ordnung der Entwicklung: Zur Ethnographie der Entwicklungspraxis und ihrer ethischen Implikationen, Geogr. Z., 92, 208-226, 2004.

McEwan, C.: Postcolonialism and Developement, Routledge, New York u.a., 2009.

Menzel, U.: Das Ende der Dritten Welt und das Scheitern der großen Theorie, Suhrkamp, Frankfurt/M., 1992.
Müller-Mahn, D. und Verne, J.: Geographische Entwicklungsforschung - alte Probleme, neue Perspektiven, Geographische Rundschau, 62, 4-11, 2010.

Power, M.: Rethinking Development Geographies, London, Routledge, New York, 2003.

Radcliffe, S.: Development and geography: towards a postcolonial development geography?, Progess in Human Geography, 29, 291-298, 2005.

Rahnema, M. und Bawtree, V. (Hrsg.): The Post-Development Reader, Zed Books, London, 1997.

Rist, G.: The History of Development. From Western Origins to Global Faith, Zed Books, London, 1997.

Sachs, W. (Hrsg.): The Development Dictionary. A Guide to Knowledge as Power, Zed Books, London, 1992.

Spivak, G. C.: The Post-Colonial Critic: interviews, strategies, dialogues, Routledge, London, 1990. 